九州大学学術情報リポジトリ

Kyushu University Institutional Repository

\title{
Infinite variation tempered stable Ornstein- Uhlenbeck processes with discrete observations
}

Kawai, Rei ichiro

Department of Mathematics, University of Leicester

Masuda, Hiroki

Faculty of Mathematics, Kyushu University

http://hdl. hand le. net/2324/16248

出版情報 : MI Preprint Series. 2010-2，2010-01-17. 九州大学大学院数理学研究院 バージョン：

権利関係 : 


\title{
MI Preprint Series
}

Kyushu University

The Global COE Program

Math-for-Industry Education \& Research Hub

\section{Infinite variation tempered stable Ornstein-Uhlenbeck processes with discrete observations}

\author{
Reiichiro Kawai \\ \& Hiroki Masuda
}

MI 2010-2

( Received January 17, 2010 )

Faculty of Mathematics

Kyushu University

Fukuoka, JAPAN 


\title{
Infinite Variation Tempered Stable Ornstein-Uhlenbeck Processes with Discrete Observations
}

\author{
REIICHIRO KAWAI*AND HIROKI MASUDA ${ }^{\dagger}$
}

\begin{abstract}
We investigate transition law between consecutive observations of Ornstein-Uhlenbeck processes of infinite variation with tempered stable stationary distribution. Thanks to the Markov autoregressive structure, the transition law can be written in the exact sense as a convolution of three random components; a compound Poisson distribution and two independent tempered stable distributions, one with stability index in $(0,1)$ and the other with index in $(1,2)$. We discuss simulation techniques for those three random elements. With the exact transition law and proposed simulation techniques, sample paths simulation proves significantly more efficient, relative to the known approximative technique based on infinite shot noise series representation of tempered stable Lévy processes.

Keywords: acceptance-rejection sampling, Lévy process, Ornstein-Uhlenbeck processes, selfdecomposability, transition law, tempered stable process.
\end{abstract}

2010 Mathematics Subject Classification: 60J75, 62E15, 65C10, 68U20.

\section{Introduction}

The class of non-Gaussian Ornstein-Uhlenbeck (OU, in short) processes is closely related to the selfdecomposability of the infinitely divisible distribution. Several interesting properties are known, such as the explicit relation between Lévy measures of the stationary distribution and the underlying Lévy process and the representation of entire trajectory based on shot noise series representation of Lévy processes, to mention just a few. (For details, see Section 17 of Sato [14],

\footnotetext{
This version: January 17, 2010.

*Email Address: reiichiro.kawai@gmail.com. Postal Address: Department of Mathematics, University of Leicester, Leicester LE1 7RH, UK.

${ }^{\dagger}$ Email Address: hiroki@math.kyushu-u.ac.jp. Postal Address: Graduate School of Mathematics, Kyushu University, Fukuoka 819-0395, Japan.
} 
Masuda [10] and references therein.) Also, due to the growing practical interest, many authors have proposed statistical inference methods for non-Gaussian OU processes. (See, for example, Brockwell et al. [4], Jongbloed et al. [8] and Sun and Zhang [15].)

In the class of non-Gaussian OU processes, the class of tempered stable OU (TS-OU, in short) process of finite variation has been of particular interest from both theoretical and practical points of view. In terms of mathematical tractability, the transition law between consecutive observations can be written in the exact sense as a convolution of one compound Poisson and one tempered stable distributions. It is known that exact, yet simple, simulation methods are available for both random elements. The particular setting of inverse-Gaussian OU processes was studied in Zhang and Zhang [17], while the general setting in [9]. Also, it was shown in Zhang and Zhang [18] that the transition law is selfdecomposable when the stability index is no less than $1 / 2$. In practice, due to its distributional flexibility and the positivity of sample paths, they have been used in financial economics and mathematical finance (for example, Barndorff-Nielsen and Shephard [2] and Benth et al. [3]).

In this paper, we study the class of TS-OU processes of infinite variation, that is, OU processes with a tempered stable stationary distribution with stability index in $(1,2)$. This can be thought of as a natural alternative of finite variation TS-OU processes, while the extension is not straightforward. In fact, the structure of transition law turns out to be significantly different, in the sense that for example, the transition law is a convolution of two independent tempered stable and one compound Poisson components. Also, the support of sample paths is necessarily the whole real line, while only the positive half line in the finite variation setting if no negative jumps exist. We will here only dealt with a unilateral setting with no negative jumps. Nevertheless, the bilateral setting is also within our scope as it can be treated simply by superpositioning another similar convolution of three independent random components. In addition, the bilateral framework can produce more distributional flexibility through combinations of positive and negative jump components in terms of, for example, stability index and even finite and infinite variations. They may widen the applicability of OU processes in a variety of fields.

The rest of this paper is organized as follows. Section $\square$ summarizes background material on stable and tempered stable distributions and on OU processes with tempered stable stationary distribution. In Section B, we derive the transition law in closed form, consisting of three random components; a compound Poisson distribution and two independent tempered stable distributions, one with stability index $\alpha \in(1,2)$, while the other with index $\alpha-1 \in(0,1)$. In Section 4 , we discuss simulation methods for the three random elements, all of which are based on acceptancerejection sampling techniques. We also provide numerical results to illustrate the effectiveness of our exact transition law and proposed simulation techniques in sample paths generation, relative to the existing approximative method with infinite shot noise series representation of tempered stable 
Lévy processes. Finally, Section [5 concludes.

\section{Preliminaries}

Let us begin this preliminary section with the notations which will be used throughout the paper. We denote by $\mathbb{R}$ the one dimensional Euclidean space with the norm $|\cdot|$ and $\mathbb{R}_{+}:=(0,+\infty)$. Let $\mathbb{N}$ be the collection of positive integers with $\mathbb{N}_{0}:=\mathbb{N} \cup\{0\}$. We denote by $\stackrel{\mathscr{L}}{=}$ the identity in law. We denote by $\Gamma(a, b)$ the gamma distribution with density $b^{a} / \Gamma(a) z^{a-1} e^{-b z}$. We write $f_{L}(z)$ for a probability density function of a distribution $L$. (For example, $f_{\Gamma(a, b)}(z)=b^{a} / \Gamma(a) z^{a-1} e^{-b z}$.) We fix $(\Omega, \mathscr{F}, \mathbb{P})$ as our underlying probability space. Finally, note that $\Gamma(-s)<0$ for $s \in(0,1)$, while $\Gamma(-s)>0$ for $s \in(1,2)$.

\subsection{Spectrally Positive Stable Processes}

Let $\left\{L_{t}^{(\mathrm{s})}: t \geq 0\right\}$ be a totally positively skewed stable (Lévy) process satisfying

$$
\begin{aligned}
\mathbb{E}\left[e^{\left.i y L_{t}^{(\mathrm{s})}\right]}\right. & =\exp \left[\operatorname{ta} \Gamma(-\alpha) \cos \left(\frac{\pi \alpha}{2}\right)|y|^{\alpha}\left(1-i \tan \frac{\pi \alpha}{2} \operatorname{sgn}(y)\right)\right] \\
& = \begin{cases}\exp \left[t \int_{\mathbb{R}_{+}}\left(e^{i y z}-1\right) \frac{a}{z^{\alpha+1}} d z\right], & \text { if } \alpha \in(0,1), \\
\exp \left[t \int_{\mathbb{R}_{+}}\left(e^{i y z}-1-i y z\right) \frac{a}{z^{\alpha+1}} d z\right], & \text { if } \alpha \in(1,2),\end{cases}
\end{aligned}
$$

with some $a>0$. Throughout this paper, we exclude the case $\alpha=1$. We write $S(\alpha, a):=\mathscr{L}\left(L_{1}^{(\mathrm{s})}\right)$. The $C^{+\infty}$-density of the distribution $S(\alpha, a)$ is given in the form of convergent power series

$$
f_{S(\alpha, b)}(x)= \begin{cases}\frac{(-a \Gamma(-\alpha))^{-1 / \alpha}}{\pi} \sum_{k=1}^{+\infty}(-1)^{k-1} \sin (\pi k \alpha) \frac{\Gamma(k \alpha+1)}{k !}\left(\frac{x}{(-a \Gamma(-\alpha))^{1 / \alpha}}\right)^{-k \alpha-1}, & \text { if } \alpha \in(0,1), \\ \frac{(a \Gamma(-\alpha))^{-1 / \alpha}}{\pi} \sum_{k=1}^{+\infty} \sin \left(\pi k \frac{1-\alpha}{\alpha}\right) \frac{\Gamma(k / \alpha+1)}{k !}\left(-\frac{x}{(a \Gamma(-\alpha))^{1 / \alpha}}\right)^{k-1}, & \text { if } \alpha \in(1,2) .\end{cases}
$$

Note that the above density is defined on $\mathbb{R}_{+}$if $\alpha \in(0,1)$, while on $\mathbb{R}$ if $\alpha \in(1,2)$. It holds that for each $t>0, \mathscr{L}\left(L_{t}^{(\mathrm{s})}\right)=S(\alpha, t a)$, and by the scaling property, $\mathscr{L}\left(t^{-1 / \alpha} L_{t}^{(\mathrm{s})}\right)=S(\alpha, a)$. Note that the distribution $S(\alpha, t a)$ has density $t^{-1 / \alpha} f_{S(\alpha, a)}\left(t^{-1 / \alpha} x\right)$. The distribution $S(\alpha, a)$ can be simulated in the exact sense through the well known representation, due to Chambers et al. [5],

$$
S(\alpha, a) \stackrel{\mathscr{L}}{=}(-a \Gamma(-\alpha) \cos (\pi \alpha / 2))^{1 / \alpha} \frac{\sin (\alpha V+\theta)}{(\cos V \cos \theta)^{1 / \alpha}}\left(\frac{\cos ((1-\alpha) V-\theta)}{E}\right)^{\frac{1-\alpha}{\alpha}}
$$

where $\theta:=\arctan (\tan (\pi \alpha / 2)), V$ is a uniform random variable on $(-\pi / 2, \pi / 2)$ and $E$ is a standard exponential random variable independent of $V$. See Zolotarev [19] for more details on the stable distribution. 


\subsection{Spectrally Positive Tempered Stable Processes}

Let $\left\{L_{t}^{(\mathrm{ts})}: t \geq 0\right\}$ be a centered and totally positively skewed tempered stable (Lévy) process satisfying

$$
\mathbb{E}\left[e^{i y L_{t}^{(\mathrm{ts})}}\right]=\exp \left[t \int_{\mathbb{R}_{+}}\left(e^{i y z}-1-i y z\right) a \frac{e^{-b z}}{z^{\alpha+1}} d z\right]=\exp \left[\operatorname{ta} \Gamma(-\alpha)\left((b-i y)^{\alpha}-b^{\alpha}+i y \alpha b^{\alpha-1}\right)\right] .
$$

Note that as indicated by "centered", the tempered stable process here is centered even in the case $\alpha \in(0,1)$, unlike in the case of the stable process characterized by (2.1). As a matter of course, when $\alpha \in(0,1)$, by adding back the centering term as $L_{t}^{(\mathrm{ts})}+t \Gamma(1-\alpha) a b^{\alpha-1}$, we can recover the associated tempered stable subordinator. Throughout the paper, we will use the notations

$$
T S(\alpha, a, b):=\mathscr{L}\left(L_{1}^{(\mathrm{ts})}\right)
$$

and

$$
T S^{\prime}(\alpha, a, b):=\mathscr{L}\left(L_{1}^{(\mathrm{ts})}+\Gamma(1-\alpha) a b^{\alpha-1}\right) .
$$

It is known that

$$
\frac{e^{-b z}}{\mathbb{E}\left[e^{\left.-b L_{1}^{(\mathrm{s})}\right]}\right.} f_{S(\alpha, a)}(z)=e^{-b z-a \Gamma(-\alpha) b^{\alpha}} f_{S(\alpha, a)}(z)=f_{T S^{\prime}(\alpha, a, b)}(z)
$$

The class of tempered stable distributions was first proposed by Tweedie [16]. Several featuring properties of tempered stable distributions and processes were revealed by Rosiński [13], such as a stable-like behavior over short intervals, the absolute continuity with respect to its short-range limiting stable process, an aggregational Gaussianity and an infinite shot noise series representation in closed form

$$
\begin{aligned}
\left\{L_{t}^{(\mathrm{ts})}: t \in[0, T]\right\} \stackrel{\mathscr{L}}{=}\{ & \sum_{k=1}^{+\infty}\left[\left[\left(\frac{\alpha \Gamma_{k}}{T a}\right)^{-1 / \alpha} \wedge \frac{V_{k} U_{k}^{1 / \alpha}}{b}\right] \mathbb{1}_{[0, t]}\left(T_{k}\right)-\frac{t}{T}\left(\frac{\alpha k}{T a}\right)^{-1 / \alpha} \mathbb{1}_{(1,2)}(\alpha)\right] \\
& \left.+\frac{t}{T}\left(\frac{T a}{\alpha}\right)^{1 / \alpha} \zeta(1 / \alpha) \mathbb{1}_{(1,2)}(\alpha)-t \Gamma(1-\alpha) a b^{\alpha-1}: t \in[0, T]\right\},
\end{aligned}
$$

where $\left\{\Gamma_{k}\right\}_{k \in \mathbb{N}}$ are arrival times of a standard Poisson process, $\left\{T_{k}\right\}_{k \in \mathbb{N}}$ is a sequence of iid uniform random variables on $[0, T],\left\{V_{k}\right\}_{k \in \mathbb{N}}$ is a sequence of iid standard exponential random variables and $\left\{U_{k}\right\}_{k \in \mathbb{N}}$ is a sequence of iid uniform random variables on [0,1]. All those random sequences are mutually independent. Note that the kernel of series representation is not unique. In fact, there are a different series representation derived in Imai and Kawai [7] through the thinning and rejection methods and yet another representation numerically through the inverse Lévy measure method. (For details about the methods, see Rosiński [12].) 


\subsection{Ornstein-Uhlenbeck Processes with Tempered Stable Stationary Distri- bution}

Consider the stochastic process $\left\{X_{t}: t \geq 0\right\}$ defined in form of stochastic differential equation

$$
d X_{t}=\lambda\left(\mu-X_{t}\right) d t+d Z_{\lambda t}
$$

where $\lambda>0, \mu \in \mathbb{R}$ and $\left\{Z_{t}: t \geq 0\right\}$ is a Lévy process (not necessarily a subordinator), or in canonical form

$$
X_{t}=e^{-\lambda t} X_{0}+\mu\left(1-e^{-\lambda t}\right)+\int_{0}^{t} e^{-\lambda(t-s)} d Z_{\lambda s} .
$$

Processes of this type are often called non-Gaussian OU processes, or Lévy-driven OU processes. With $\left\{Z_{t}: t \geq 0\right\}$ being a subordinator, they have been used, for example, to model the squared volatility in a stochastic volatility model of Barndorff-Nielsen and Shephard [2], due to the nonnegativity of sample paths.

In this paper, we consider the class of OU processes (2.8) where its invariant law $\mathscr{L}\left(\lim _{t \uparrow+\infty} X_{t}\right)$ is $T S(\alpha, a, b) * \delta_{\mu}$ with $\alpha \in(1,2)$, where $*$ denotes the convolution and $\delta_{\mu}$ is the degenerate distribution at $\mu$. The invariant law is clearly self-decomposable and has Lévy density

$$
u(z)=a \frac{e^{-b z}}{z^{\alpha+1}}, \quad z \in \mathbb{R}_{+} .
$$

In fact, since the law has finite moments of every order due to the exponential tempering in (2.10), it follows that regardless of the choice of the parameter $\lambda>0$, there exists an ergodic Lévy-driven OU process having $T S(\alpha, a, b) * \delta_{\mu}$ as its invariant law. (We refer the reader to Masuda [10] and the references therein for details about Lévy-driven OU processes.) In particular, OU process with inverse Gaussian invariant law $(\alpha=1 / 2)$ was applied in Benth [3] to stochastic volatility modeling of [2] for volatility and variance swap valuations.

Let $w(z)$ be the Lévy density of the marginal $Z_{1}$ of the background driving Lévy process. Since $u(z)$ is differentiable, the Lévy densities $w(z)$ and $u(z)$ are related by

$$
w(z)=-u(z)-z \frac{\partial}{\partial z} u(z)=a \alpha \frac{e^{-b z}}{z^{\alpha+1}}+a b \frac{e^{-b z}}{z^{(\alpha-1)+1}} .
$$

Therefore, on the one hand, if $\alpha \in(0,1)$, then the underlying process $\left\{Z_{t}: t \geq 0\right\}$ is the superposition of a tempered stable process with $T S^{\prime}(\alpha, a \alpha, b)$ and a compound Poisson process with Lévy density $a b z^{-\alpha} e^{-b z}$. Sample paths can be written in the exact sense, using the infinite shot 
noise series representation $(2.7)$ as

$$
\begin{aligned}
\left\{X_{t}: t \in[0, T]\right\} \stackrel{\mathscr{L}}{=}\left\{e^{-\lambda t} X_{0}+\mu\left(1-e^{-\lambda t}\right)+\right. & \sum_{k=1}^{+\infty} e^{-\lambda\left(t-T_{k}\right)}\left[\left(\frac{\Gamma_{k}}{a T}\right)^{-1 / \alpha} \wedge \frac{V_{k} U_{k}^{1 / \alpha}}{b}\right] \mathbb{1}_{[0, t]}\left(T_{k}\right) \\
& \left.+\sum_{k=1}^{+\infty} e^{\widetilde{\Gamma}_{k}-\lambda t} G_{k} \mathbb{1}_{[0, \lambda t]}\left(\widetilde{\Gamma}_{k}\right): t \in[0, T]\right\},
\end{aligned}
$$

where $\left\{\widetilde{\Gamma}_{k}\right\}_{k \in \mathbb{N}}$ are arrival times of a standard Poisson process, independent of $\left\{\Gamma_{k}\right\}_{k \in \mathbb{N}}$, with intensity $\Gamma(1-\alpha) a b^{\alpha}\left(=\int_{\mathbb{R}_{+}} a b z^{-\alpha} e^{-b z} d z\right)$, and $\left\{G_{k}\right\}_{k \in \mathbb{N}}$ is a sequence of iid random variables with gamma distribution $\Gamma(1-\alpha, b)$.

On the other hand, if $\alpha \in(1,2)$, then the equation (2.II) implies that the underlying process $\left\{Z_{t}: t \geq 0\right\}$ is a superposition of two independent tempered stable processes with $T S(\alpha, a \alpha, b)$ and $T S(\alpha-1, a b, b)$. Sample paths can also be written in the exact sense with infinite shot noise series representation (2.7). This is however not very sensible, at least for the following three reasons; (i) there are too many random sequences to be generated, (ii) the series representation for $\operatorname{TS}(\alpha, a \alpha, b)$ contains intricate centering terms as seen in ([2.7), and (iii) the issue of truncation error has to be addressed for two infinite shot noise series.

\section{Transition Law of Tempered Stable Ornstein-Uhlenbeck Pro- cesses of Infinite Variation}

In this section, we derive the transition law between consecutive observations of discrete time skeleton

$$
X_{0}, X_{\Delta}, X_{2 \Delta}, \cdots
$$

of infinite variation TS-OU processes (2.8), with a fixed time stepsize $\Delta>0$. (In principle, the stepsize does not need to be equidistant and can be set different positive values for different steps.) The difference from the finite variation setting [18, 9] lies in the integrability of Lévy density of the transition law around the origin. As a consequence, the Lévy density has to be decomposed twice to extract all infinite activity part, while only once in the finite variation case. For better 
presentation, we will use the following notations

$$
\begin{aligned}
w_{1, \Delta}(z) & :=\left(1-e^{-\alpha \lambda \Delta}\right) \frac{a}{z^{\alpha+1}} e^{-b z}, \\
w_{2, \Delta}(z) & :=e^{-\alpha \lambda \Delta} \frac{a}{z^{\alpha+1}}\left(e^{-b z}-e^{-b e^{\lambda \Delta} z}\right), \\
w_{21, \Delta}(z) & :=e^{-\alpha \lambda \Delta}\left(e^{\lambda \Delta}-1\right) \frac{a b}{z^{(\alpha-1)+1}} e^{-b e^{\lambda \Delta} z}, \\
w_{22, \Delta}(z) & :=a e^{-\alpha \lambda \Delta} \frac{e^{-b z}-e^{-b e^{\lambda \Delta} z}-b\left(e^{\lambda \Delta}-1\right) z e^{-b e^{\lambda \Delta} z}}{z^{\alpha+1}} .
\end{aligned}
$$

Theorem 3.1. Fix $\Delta>0$. For each $n \in \mathbb{N}_{0}$, it holds that given $X_{n \Delta}$,

$$
X_{(n+1) \Delta} \stackrel{\mathscr{L}}{=} e^{-\lambda \Delta} X_{n \Delta}+\mu\left(1-e^{-\lambda \Delta}\right)+Y_{01}+Y_{02}+\left(\sum_{k=1}^{N_{\kappa_{\Delta}}} \Theta_{k}-\Delta \gamma\right)
$$

where

$$
\gamma:=a b^{\alpha-1} \Gamma(1-\alpha)\left(e^{-\alpha \lambda \Delta}-e^{-\lambda \Delta}-\left(e^{-\lambda \Delta}-e^{-2 \lambda \Delta}\right)(1-\alpha)\right)
$$

and all the random elements are mutually independent and specified as

- $Y_{01} \sim T S\left(\alpha, a\left(1-e^{-\alpha \lambda \Delta}\right), b\right)$,

- $Y_{02} \sim T S\left(\alpha-1, a b e^{-\alpha \lambda \Delta}\left(e^{\lambda \Delta}-1\right), b e^{\lambda \Delta}\right)$,

- $\quad N_{\kappa_{\Delta}} \sim \operatorname{Pois}\left(\kappa_{\Delta}\right)$ with $\kappa_{\Delta}:=a b^{\alpha} \Gamma(-\alpha)\left(\alpha\left(1-e^{-\lambda \Delta}\right)+e^{-\alpha \lambda \Delta}-1\right)$,

- $\left\{\Theta_{k}\right\}_{k \in \mathbb{N}}$ is a sequence of iid random variables in $\mathbb{R}_{+}$with common probability density $v_{1, \Delta}(z):=\kappa_{\Delta}^{-1} w_{22, \Delta}(z)$.

Moreover, the transition law is selfdecomposable.

Proof. By the homogeneous Markovian autoregressive structure of (2.9), it holds that for each $n \in \mathbb{N}_{0}$, given $X_{n \Delta}$,

$$
\begin{aligned}
X_{(n+1) \Delta} & =e^{-\lambda \Delta} X_{n \Delta}+\mu\left(1-e^{-\lambda \Delta}\right)+\int_{n \Delta}^{(n+1) \Delta} e^{-\lambda((n+1) \Delta-s)} d Z_{\lambda s} \\
& =: e^{-\lambda \Delta} X_{n \Delta}+\mu\left(1-e^{-\lambda \Delta}\right)+\varepsilon_{\Delta, n+1} \\
& \mathscr{L}=e^{-\lambda \Delta} X_{n \Delta}+\mu\left(1-e^{-\lambda \Delta}\right)+\int_{0}^{\lambda \Delta} e^{-\lambda \Delta+s} d Z_{s},
\end{aligned}
$$

where the identity in law holds by the independence and stationarity of increments of the underlying Lévy process $\left\{Z_{t}: t \geq 0\right\}$. This implies that $\left\{\varepsilon_{\Delta, k}\right\}_{k \in \mathbb{N}}$ reduces to a sequence of iid random variables with common distribution $F_{\Delta}:=\mathscr{L}\left(\int_{0}^{\lambda \Delta} e^{-\lambda \Delta+s} d Z_{s}\right)$. It thus suffices to investigate the conditional law $\mathscr{L}\left(X_{\Delta} \mid X_{0}\right)$, that is, only of the first increment. Note that by definition, this law is infinitely divisible. 
Using the Lévy-integral transform, we get the characteristic function of the distribution $F_{\Delta}$ as

$$
\widehat{F}_{\Delta}(y)=\exp \left[\int_{\mathbb{R}_{+}}\left(e^{i y z}-1-i y z\right) w_{\Delta}(z) d z\right],
$$

where

$$
w_{\Delta}(z):=\int_{0}^{\lambda \Delta} e^{s} w\left(e^{s} z\right) d s
$$

By further computing $w_{\Delta}(z)$, we get

$$
\begin{aligned}
w_{\Delta}(z) & =\frac{a}{z^{\alpha+1}} \int_{0}^{\lambda \Delta}\left(\alpha+b e^{s} z\right) e^{-\alpha s} e^{-b e^{s} z} d s \\
& =\frac{a}{z^{\alpha+1}} \int_{0}^{\lambda \Delta} \frac{\partial}{\partial s}\left(e^{-\alpha s} e^{-b e^{s} z}\right) d s \\
& =\frac{a}{z^{\alpha+1}}\left(e^{-b z}-e^{-\alpha \lambda \Delta-b e^{\lambda \Delta} z}\right) \\
& =w_{1, \Delta}(z)+w_{2, \Delta}(z) .
\end{aligned}
$$

Note that $w_{1, \Delta}$ and $w_{2, \Delta}$ are positive functions on $\mathbb{R}_{+}$. Clearly, $w_{1, \Delta}$ is the smooth Lévy density of $T S\left(\alpha,\left(1-e^{-\alpha \lambda \Delta}\right) a, b\right)$. Note that $w_{2, \Delta}(z) \sim a b e^{-\alpha \lambda \Delta}\left(e^{\lambda \Delta}-1\right) z^{-\alpha}$ as $z \downarrow 0$. This implies that $w_{2, \Delta}$ is not integrable and thus cannot be a Lévy density of compound Poisson components.

Let us further decompose $w_{2, \Delta}$ into two parts. We use the identity

$$
e^{-x}-e^{-y}=(y-x) e^{-y}+e^{-x}(y-x)^{2} H(y-x), \quad y>x>0 .
$$

where $H(z):=z^{-2}\left(1-e^{-z}(1+z)\right)$ is positive, bounded and strictly decreasing on $\mathbb{R}_{+}$such that $\lim _{z \downarrow 0} H(z)=1 / 2$. By applying this identity, we get

$$
\begin{aligned}
w_{2, \Delta}(z)= & e^{-\alpha \lambda \Delta}\left(e^{\lambda \Delta}-1\right) \frac{a b}{z^{(\alpha-1)+1}} e^{-b e^{\lambda \Delta} z} \\
& +a b^{2}\left(e^{\lambda \Delta}-1\right)^{2} e^{-\alpha \lambda \Delta} z^{(2-\alpha)-1} e^{-b z} H\left(b\left(e^{\lambda \Delta}-1\right) z\right) \\
= & w_{21, \Delta}(z)+w_{22, \Delta}(z) .
\end{aligned}
$$

Clearly, $w_{21, \Delta}$ is the smooth Lévy density of $\operatorname{TS}\left(\alpha-1, a b e^{-\alpha \lambda \Delta}\left(e^{\lambda \Delta}-1\right), b e^{\lambda \Delta}\right)$.

Next, observe that

$$
\int_{\mathbb{R}_{+}} w_{22, \Delta}(z) d z=a e^{-\alpha \lambda \Delta} \int_{\mathbb{R}_{+}} z^{-1-\alpha}\left(e^{-b z}-e^{-b e^{\lambda \Delta_{z}}}-b\left(e^{\lambda \Delta}-1\right) z e^{-b e^{\lambda \Delta_{z}}}\right) d z=\kappa_{\Delta} .
$$

This shows that $w_{22, \Delta}$ serves as a Lévy density of compound Poisson components. To realize the centering for the compound Poisson distribution due to (B.2), we need to subtract the constant term $\int_{\mathbb{R}_{+}} z w_{22, \Delta}(z) d z=\gamma$, multiplied by the time stepsize $\Delta$. 
It remains to show the selfdecomposability of the transition law. Define

$$
h_{\Delta}(z):=\frac{1}{z^{\alpha}} \int_{0}^{\lambda \Delta}\left(a+b e^{s} z\right) e^{-\alpha s-b e^{s} z} d s, \quad z \in \mathbb{R}_{+},
$$

so that $w_{\Delta}(z)=a h_{\Delta}(z) / z$. This function is obviously non-negative and is decreasing on $\mathbb{R}_{+}$, due to

$$
\begin{aligned}
\frac{d}{d z} h_{\Delta}(z) & =\int_{0}^{\lambda \Delta} z^{-1-\alpha} e^{-\alpha s-b e^{s} z}\left(-\left(b e^{s}\right)^{2} z^{2}-(2 \alpha-1) b e^{s} z-\alpha^{2}\right) d s \\
& \leq-\frac{\alpha^{2}}{z^{1+\alpha}} \int_{0}^{\lambda \Delta} e^{-\alpha s-b e^{s} z} d s<0 .
\end{aligned}
$$

Hence, Corollary 15.11 of Sato [14] yields the claim. The proof is complete.

Remark 3.2. It is worth noting that the selfdecomposability of the transition law holds for any $\alpha \in(1,2)$ in the infinite variation setting, while in the finite variation case, it holds only for the stability index of no less than $1 / 2$. This difference occurs due to the term $2 \alpha-1$ in the integrand of (B.4), that is, the sign of $2 \alpha-1$ changes at $\alpha=1 / 2$. (See Zhang and Zhang [18] for the finite variation case.)

Remark 3.3. It is difficult to provide an efficient simulation method for the distribution induced by the Lévy density $w_{2, \Delta}$. Nevertheless, in the finite variation setting, that is, if $\alpha \in(0,1)$, then $w_{2, \Delta}$ is integrable

$$
\int_{\mathbb{R}_{+}} w_{2, \Delta}(z) d z=-a b^{\alpha} \Gamma(-\alpha)\left(1-e^{-\alpha \lambda \Delta}\right) \in \mathbb{R}_{+} .
$$

This shows that $w_{2, \Delta}$ acts as the smooth Lévy density of a compound Poisson distribution. Moreover, there is no further need to decompose $w_{2, \Delta}$, unlike we did in Theorem B.1, since the corresponding Poisson distribution can be simulated in the exact sense through an acceptance-rejection sampling method, which will be presented in Section 4.1.

\section{Simulation Methods}

It follows from Theorem 3.$]$ that to simulate sample paths of infinite variation TS-OU processes at discrete timings, it suffices to simulate three random elements, that is, the tempered stable random variables $Y_{01}$ and $Y_{02}$, and the compound Poisson random variable $\sum_{k=1}^{N_{\kappa_{\Delta}}} \Theta_{k}$. In this section, we discuss simulation methods for those random elements. We begin with relatively straightforward cases of $Y_{02}$ and of the compound Poisson $\sum_{k=1}^{N_{\kappa_{\Delta}}} \Theta_{k}$. 


\subsection{Simulation of Tempered Stable with Stability Index $\alpha-1$}

First, we present an exact simulation method for $Y_{02} \sim T S\left(\alpha-1, a b e^{-\alpha \lambda \Delta}\left(e^{\lambda \Delta}-1\right), b e^{\lambda \Delta}\right)$, which is centered. Hence, we will first instead simulate $\operatorname{TS}^{\prime}\left(\alpha-1, a b e^{-\alpha \lambda \Delta}\left(e^{\lambda \Delta}-1\right), b e^{\lambda \Delta}\right)$, that is,

$$
Y_{02}^{\prime}:=Y_{02}+\Gamma(2-\alpha) a b^{\alpha}\left(1-e^{-\lambda \Delta}\right)
$$

which takes values solely in $\mathbb{R}_{+}$and then subtract the added constant term. An efficient and exact simulation method for the case $\alpha-1=0.5$, that is the inverse Gaussian, is well known due to Michael et al. [1]]. For the general case of $\alpha-1 \in(0,1)$, the best route would be acceptancerejection sampling based on the representation $([2.3)$ of the stable distribution and the likelihood ratio of the two densities; for each $z \in \mathbb{R}_{+}$,

$$
\frac{f_{T S^{\prime}\left(\alpha-1, a b e^{-\alpha \lambda \Delta}\left(e^{\lambda \Delta}-1\right), b e^{\lambda \Delta}\right)}(z)}{f_{S\left(\alpha-1, a b e^{-\alpha \lambda \Delta}\left(e^{\lambda \Delta_{-}}\right)\right)}(z)}=e^{-b e^{\lambda \Delta_{z}}-\Gamma(1-\alpha) a b^{\alpha+1}\left(e^{\lambda \Delta}-1\right)} \leq e^{-\Gamma(1-\alpha) a b^{\alpha+1}\left(e^{\lambda \Delta}-1\right)},
$$

where the density functions $f_{S(\alpha, a)}$ and $f_{T S^{\prime}(\alpha, a, b)}$ are given respectively by (2.2) and ([2.6). The random variable $Y_{02}$ can then be generated in the exact sense by the following simple acceptancerejection sampling algorithm.

$\operatorname{Algorithm} 1\left(Y_{02} \sim T S\left(\alpha-1, a b e^{-\alpha \lambda \Delta}\left(e^{\lambda \Delta}-1\right), b e^{\lambda \Delta}\right)\right)$ :

Step 1. Generate $U$ as uniform $(0,1)$ and $V$ as $S\left(\alpha-1, a b e^{-\alpha \lambda \Delta}\left(e^{\lambda \Delta}-1\right)\right)$ through the representation (2.3)).

Step 2. If $U \leq e^{-b e^{\lambda \Delta} V}$, let $Y_{02} \leftarrow V-\Gamma(2-\alpha) a b^{\alpha}\left(1-e^{-\lambda \Delta}\right)$. Otherwise, return to Step 1 .

The acceptance rate at Step 2 of Algorithm 1 is given by

$$
p_{1}(\Delta):=\mathbb{P}\left(U \leq e^{-b e^{\lambda \Delta} V}\right)=e^{\Gamma(1-\alpha) a b^{\alpha+1}\left(e^{\lambda \Delta}-1\right)} .
$$

Clearly, the algorithm works more efficiently when the acceptance rate $p_{1}(\Delta)$ at Step 2 is closer to 1. Indeed, this happens when $\Delta \downarrow 0$.

It is, however, more practical to discuss the effectiveness on the work-normalized basis. Since the simulation of $\mathscr{L}\left(Y_{02}\right)$ is exact through Algorithm 1, all we need to pay attention to is the computing time required to generate iid increments from $\operatorname{TS}\left(\alpha-1, a b e^{-\alpha \lambda \Delta}\left(e^{\lambda \Delta}-1\right), b e^{\lambda \Delta}\right)$ to fill each sample path. Since we are concerned with sample paths over a finite time horizon, by taking a smaller time stepsize $\Delta$, the number of increments for each sample path increases in proportion to $1 / \Delta$. Next, in Algorithm 1, the number of trials until one acceptance has the geometric distribution with success probability $p_{1}(\Delta)$. The average time to get one sample from Algorithm 1 is thus proportional to $1 / p_{1}(\Delta)$. Then, we find that as $\Delta \downarrow 0$,

$$
\frac{1}{\Delta \cdot p_{1}(\Delta)} \simeq \frac{1}{\Delta},
$$


which implies that the average computing time related to $Y_{02}$ for each sample path increases in proportion to $1 / \Delta$ as $\Delta \downarrow 0$.

For more details about related acceptance-rejection sampling methods, see Baeumer and Meerschaert [1], Devroye [6] and Kawai and Masuda [9].

\subsection{Simulation of Compound Poisson Component}

Next, we consider simulation of the compound Poisson component. Generation of the Poisson random variable $N_{\kappa_{\Delta}}$ is straightforward and is thus omitted. We concentrate on generation of the random sequence $\left\{\Theta_{k}\right\}_{k \in \mathbb{N}}$. Recall that $\mathscr{L}\left(\Theta_{1}\right)$ has a probability density function $v_{1, \Delta}(z)$ and that the function $H$ in (B.3) is positive, bounded and strictly decreasing on $\mathbb{R}_{+}$with $\lim _{z \downarrow 0} H(z)=1 / 2$. We can thus show that

$$
v_{1, \Delta}(z) \leq \frac{1}{2 \kappa_{\Delta}} a b^{\alpha}\left(e^{\lambda \Delta}-1\right)^{2} e^{-\alpha \lambda \Delta} \Gamma(2-\alpha) f_{\Gamma(2-\alpha, b)}(z)=: g_{\Delta}(z),
$$

where $f_{\Gamma(2-\alpha, b)}(z)=b^{2-\alpha} \Gamma(2-\alpha)^{-1} z^{(2-\alpha)-1} e^{-b z}$ defined on $\mathbb{R}_{+}$. Then, it holds that

$$
\frac{v_{1, \Delta}(z)}{g_{\Delta}(z)}=\frac{2}{\left(e^{\lambda \Delta}-1\right)^{2} b^{2}} \frac{e^{-b z}-e^{-b e^{\lambda \Delta} z}-b\left(e^{\lambda \Delta}-1\right) z e^{-b e^{\lambda \Delta} z}}{z^{2}}=: v_{2, \Delta}(z), \quad z \in \mathbb{R}_{+} .
$$

This suggests the following acceptance-rejection sampling algorithm for generation of the random variable $\Theta_{1}$.

Algorithm $2\left(\Theta_{1}\right.$ with probability density $\left.v_{1, \Delta}(z)\right)$

Step 1: Generate $U$ as uniform $(0,1)$ and $V$ as $\Gamma(2-\alpha, b)$.

Step 2: If $U \leq v_{2, \Delta}(V)$, let $\Theta_{1} \leftarrow V$. Otherwise, return to Step 1.

The acceptance rate at Step 2 is given by

$$
p_{2}(\Delta):=\mathbb{P}\left(U \leq v_{2, \Delta}(V)\right)=\frac{2}{\alpha(\alpha-1)} \frac{\alpha\left(1-e^{-\lambda \Delta}\right)+e^{-\alpha \lambda \Delta}-1}{e^{-\alpha \lambda \Delta}\left(e^{\lambda \Delta}-1\right)^{2}} .
$$

We can show that the acceptance rate tends to 1 as $\Delta \downarrow 0$.

Since the simulation of $\left\{\Theta_{k}\right\}_{k \in \mathbb{N}}$ is exact by Algorithm 2 as well, all we need to pay attention to is the computing time required to generate iid increments from the distribution $\mathscr{L}\left(\sum_{k=1}^{N_{\kappa_{\Delta}}} \Theta_{k}\right)$ to fill each sample path. Again, by taking a smaller time stepsize $\Delta$, the number of increments over a finite time horizon increases in proportion to $1 / \Delta$. Next, the average number of implementation of Algorithm 2 required to generate one sample from the distribution $\mathscr{L}\left(\sum_{k=1}^{N_{\kappa_{\Delta}}} \Theta_{k}\right)$ is proportional 
to the intensity $\kappa_{\Delta}$ of the Poisson random variable $N_{\kappa_{\Delta}}$. The average time to get one sample from Algorithm 2 is proportional to $1 / p_{2}(\Delta)$. Therefore, we get

$$
\frac{\kappa_{\Delta}}{\Delta \cdot p_{2}(\Delta)}=\frac{a b^{\alpha} \Gamma(-\alpha) \alpha(\alpha-1) e^{-\alpha \lambda \Delta}\left(e^{\lambda \Delta}-1\right)^{2}}{2 \Delta} \simeq \Delta
$$

as $\Delta \downarrow 0$. This implies that the computing time for simulation of $\mathscr{L}\left(\sum_{k=1}^{N_{\kappa_{\Delta}}} \Theta_{k}\right)$ decreases linearly in $\Delta$, as $\Delta \downarrow 0$.

\subsection{Simulation of Tempered Stable with Stability Index $\alpha$}

We have seen that the two distributions $\mathscr{L}\left(Y_{02}\right)$ and $\mathscr{L}\left(\sum_{k=1}^{N_{\kappa_{\Delta}}} \Theta_{k}\right)$ can be simulated in the exact sense through acceptance-rejection sampling. To the best of our knowledge, the exact simulation of $Y_{01} \sim T S\left(\alpha, a\left(1-e^{-\alpha \lambda \Delta}\right), b\right)$ seems impossible in practice. For example, the series representation (2.7) looks like an exact method, while it is still approximative as soon as a finite truncation is performed. In this paper, amongst several possible approximative methods, we present a method proposed by Baeumer and Meerschaert [1]], which, we believe, is most suitable for our purpose. Its procedure is outlined as follows.

Algorithm 3 (Approximation of $\left.Y_{01} \sim T S\left(\alpha, a\left(1-e^{-\alpha \lambda \Delta}\right), b\right)\right)$ :

Fix $c>0$.

Step 1. Generate $U$ as uniform $(0,1)$ and $V$ as $S\left(\alpha, a\left(1-e^{-\alpha \lambda \Delta}\right)\right)$.

Step 2. If $U \leq e^{-b(V+c)}$, let $Y_{01, c}^{\prime} \leftarrow V$. Otherwise, return to Step 1 .

Step 3. Return $Y_{01, c} \leftarrow Y_{01, c}^{\prime}-\Gamma(1-\alpha) a\left(1-e^{-\alpha \lambda \Delta}\right) b^{\alpha-1}$.

Note that the random variable $Y_{01, c}^{\prime}$ in Step 2 approximates $T S^{\prime}\left(\alpha, a\left(1-e^{-\alpha \lambda \Delta}\right), b\right)$, while $Y_{01, c}$ approximates $T S\left(\alpha, a\left(1-e^{-\alpha \lambda \Delta}\right), b\right)$. In fact, the constant shift $-\Gamma(1-\alpha) a\left(1-e^{-\alpha \lambda \Delta}\right) b^{\alpha-1}$ in Step 3 accounts for the difference between (2.4) and (2.5)).

Let us briefly review basic properties of Algorithm 3 derived in [1]]. The acceptance rate at Step 2 of Algorithm 3 is

$$
p_{3}(\Delta, c):=\mathbb{E}\left[e^{-b(V+c)} ; V>-c\right]+\mathbb{P}(V \leq-c) .
$$

Moreover, we have

$$
\begin{aligned}
\mathbb{P}\left(Y_{01, c}^{\prime} \leq z\right) & =\frac{1}{p_{3}(\Delta, c)}\left(\mathbb{P}(V \leq \min (z,-c))+\int_{\min (z,-c)}^{z} e^{-b(y+c)} f_{S\left(\alpha, a\left(1-e^{-\alpha \lambda \Delta))}\right.\right.}(y) d y\right), \\
f_{\mathscr{L}\left(Y_{01, c}^{\prime}\right)}(z) & = \begin{cases}p_{3}(\Delta, c)^{-1} f_{S\left(\alpha, a\left(1-e^{-\alpha \lambda \Delta}\right)\right)}(z), & \text { if } z \in(-\infty,-c], \\
p_{3}(\Delta, c)^{-1} e^{-b(z+c)} f_{S\left(\alpha, a\left(1-e^{-\alpha \lambda \Delta}\right)\right)}(z), & \text { if } z \in(-c,+\infty) .\end{cases}
\end{aligned}
$$


In view of the expression

$$
f_{T S^{\prime}\left(\alpha, a\left(1-e^{-\alpha \lambda \Delta}\right), b\right)}(z)=e^{-b\left(z+a\left(1-e^{-\alpha \lambda \Delta}\right) \Gamma(-\alpha) b^{\alpha-1}\right)} f_{S\left(\alpha, a\left(1-e^{-\alpha \lambda \Delta}\right)\right)}(z), \quad z \in \mathbb{R},
$$

the parameter $c$ in Algorithm 3 acts as a truncation of the entire real line $\mathbb{R}$ to the domain on which the exponential tempering $e^{-b z}$ is performed. To sum up, we get

$$
f_{\mathscr{L}\left(Y_{01, c}\right)}(z)=f_{\mathscr{L}\left(Y_{01, c}^{\prime}\right)}\left(z+\Gamma(1-\alpha) a\left(1-e^{-\alpha \lambda \Delta}\right) b^{\alpha-1}\right), \quad z \in \mathbb{R} .
$$

It is also proved in Theorem 8 [1] that the density $f_{\mathscr{L}\left(Y_{01, c}\right)}$ converges in $L^{1}(\mathbb{R})$ to the true density $f_{T S\left(\alpha, a\left(1-e^{-\alpha \lambda \Delta), b)}\right.\right.}$ as $c \uparrow+\infty$, and consequently the Kolmogorov-Smirnov distance $D_{\mathrm{KS}}(\Delta, c)$ between two distributions $\mathscr{L}\left(Y_{01, c}\right)$ and $T S\left(\alpha, a\left(1-e^{-\alpha \lambda \Delta}\right), b\right)$ converges to zero as well. Nevertheless, it is not sensible to simply look for a smaller distribution error by taking $c \uparrow+\infty$, since then the consequent low acceptance rate makes Algorithm 3 extremely inefficient, due to $\lim _{c \uparrow+\infty} p_{3}(\Delta, c)=0$ for each $\Delta>0$. Meanwhile, it holds that for each $c>0, \lim _{\Delta \downarrow 0} p_{3}(\Delta, c)=1$. Hence, we need to address the issue of trade-off between the distribution error and the computing effort in terms of $\Delta$ and $c$.

Concerning the computing effort, on the one hand, we wish to find $(\Delta, c)$ minimizing $1 /(\Delta$. $\left.p_{3}(\Delta, c)\right)$, just as in the previous two subsections. It should be noted that asymptotic behaviors of $p_{3}(\Delta, c)$ in $\Delta$ and $c$ are difficult to analyze. Next, on the other hand, there exist several appropriate criteria to measure the distribution error. Natural candidates include $L^{1}(\mathbb{R}), L^{2}(\mathbb{R})$ distances between densities $f_{\mathscr{L}\left(Y_{01, c}\right)}$ and $f_{T S\left(\alpha, a\left(1-e^{-\alpha \lambda \Delta}\right), b\right)}$, while the Kolmogorov-Smirnov distance $D_{\mathrm{KS}}(\Delta, c)$ between the two distributions is certainly valid. However, none of them are tractable in an explicit manner. Here, for illustrative purpose, let us only present a numerical result on finding $c$ minimizing

$$
\frac{D_{\mathrm{KS}}(\Delta, c)}{\Delta \cdot p_{3}(\Delta, c)}
$$

with some fixed $\Delta>0$. Numerical results are presented in Table $\square$ with a single parameter set $(\alpha, a, b, \lambda)=(1.8,1.0,1.0,0.2)$ and $\Delta=0.1$.

It can be observed that the Kolmogorov-Smirnov distance $D_{\mathrm{KS}}(\Delta, c)$ decreases in $c$, while the acceptance rate $p_{3}(\Delta, c)$ must decrease as well. The quantity $D_{\mathrm{KS}}(\Delta, c) /\left(\Delta \cdot p_{3}(\Delta, c)\right)$ indicates that a greater value of $c$ than around 1.4 would not help in total, just as can be seen from the fact that $D_{\mathrm{KS}}(\Delta, c)$ does not improve any further, while $p_{3}(\Delta, c)$ still gets worse. We have observed from numerical experiments that the quantity $D_{\mathrm{KS}}(\Delta, c) /\left(\Delta \cdot p_{3}(\Delta, c)\right)$ behaves like a convex function in $c$. This observation is difficult to confirm in a rigorous manner, while it matches our intuition. (To find an optimal truncation point $c$, we may employ a numerical method, such as the Nelder-Mead direct search.) We draw in Figure $\square$ resulting density functions $f_{\mathscr{L}\left(Y_{01, c}\right)}$ with a few different choices 


\begin{tabular}{|c||c|c|c|}
\hline$c$ & $D_{\mathrm{KS}}(\Delta, c)$ & $p_{3}(\Delta, c)$ & $\frac{D_{\mathrm{KS}}(\Delta, c)}{\Delta \cdot p_{3}(\Delta, c)}$ \\
\hline \hline 0.0 & $1.10 \mathrm{E}-1$ & 0.875 & $1.26 \mathrm{E}+0$ \\
0.2 & $8.01 \mathrm{E}-2$ & 0.799 & $1.00 \mathrm{E}+0$ \\
0.4 & $4.74 \mathrm{E}-2$ & 0.703 & $6.73 \mathrm{E}-1$ \\
0.6 & $2.14 \mathrm{E}-2$ & 0.599 & $3.56 \mathrm{E}-1$ \\
0.8 & $7.00 \mathrm{E}-3$ & 0.499 & $1.40 \mathrm{E}-1$ \\
1.0 & $1.63 \mathrm{E}-3$ & 0.411 & $3.97 \mathrm{E}-2$ \\
1.2 & $2.69 \mathrm{E}-4$ & 0.337 & $7.98 \mathrm{E}-3$ \\
1.4 & $4.38 \mathrm{E}-5$ & 0.276 & $1.59 \mathrm{E}-3$ \\
1.6 & $4.38 \mathrm{E}-5$ & 0.226 & $1.94 \mathrm{E}-3$ \\
\hline
\end{tabular}

Table 1: Numerical results of distribution error and acceptance rate for different truncation points $c$.

of $c$, together with the true tempered stable density function $f_{T S\left(\alpha, a\left(1-e^{-\alpha \lambda \Delta}\right), b\right)}$. For better comparison, we also provide vertical lines $x=-c-\Gamma(1-\alpha) a\left(1-e^{-\alpha \lambda \Delta}\right) b^{\alpha-1}$. (We need the constant shift here because the truncation $c$ is performed on the distribution $T S^{\prime}\left(\alpha, a\left(1-e^{-\alpha \lambda \Delta}\right), b\right)$, rather than on $T S\left(\alpha, a\left(1-e^{-\alpha \lambda \Delta}\right), b\right)$.) Observe that two densities are sufficiently close when $c=1.4$.

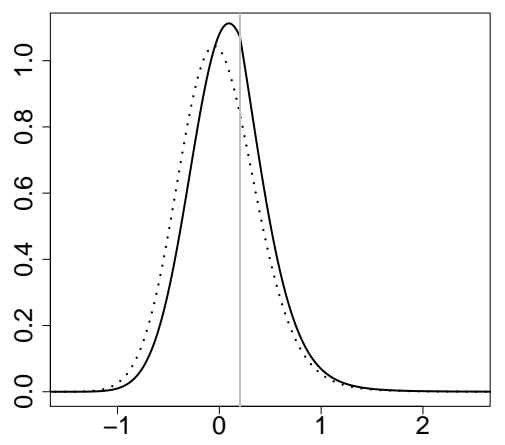

$c=0.0$

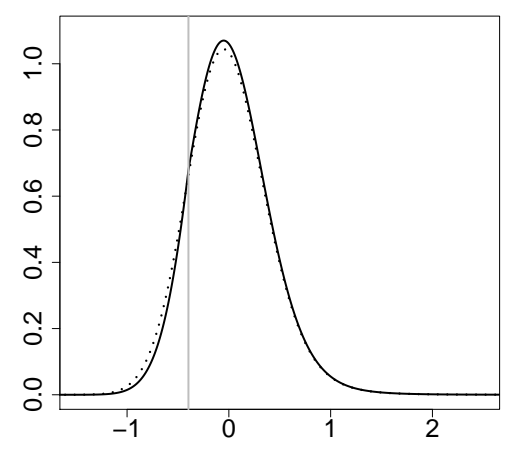

$c=0.6$

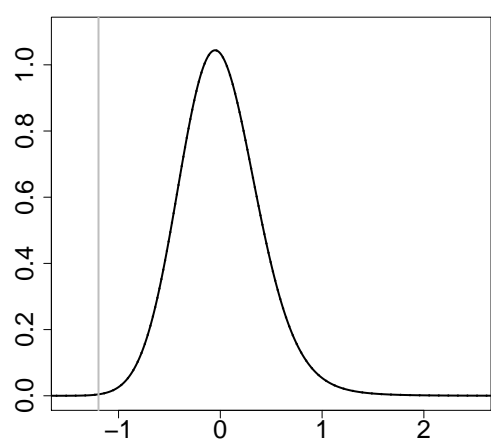

$c=1.4$

Figure 1: Comparison of two density functions $f_{\mathscr{L}\left(Y_{01, c}\right)}$ (solid) and $f_{T S\left(\alpha, a\left(1-e^{-\alpha \lambda \Delta}\right), b\right)}$ (dotted) under $(\alpha, a, b, \lambda)=(1.8,1.0,1.0,0.2)$. The horizontal line indicates $x=-c-\Gamma(1-\alpha) a(1-$ $\left.e^{-\alpha \lambda \Delta}\right) b^{\alpha-1}$.

Let us remind again that the Kolmogorov-Smirnov distance is solely one of various candidates as a measure of distribution error. The best choice of the truncation point $c$ may be different for different criteria. 


\subsection{Sample Paths}

We provide in Figure $\square$ typical sample paths of TS-OU processes of infinite variation with discrete observations, based on the transition law we have obtained in Theorem 3.1 and the simulation methods described in Algorithm 1, 2 and 3. The model parameters are set $\lambda=0.2, \mu=0, a=b=1$ and $\alpha=1.2,1.5$ and 1.8. For simplicity, we set the initial state $X_{0}=0$, that is the mean of the stationary distribution $T S(\alpha, a, b) * \delta_{\mu}$. Sample paths are generated over the time interval either $[0,100]$ or $[0,200]$, where the time stepsize is kept $\Delta=0.1$ all over in common. This means that 1000 and 2000 recursive increments are needed, respectively, for the intervals $[0,100]$ and $[0,200]$.

In our parameter setting, acceptance rates in Algorithm 1 (to generate one sample of $Y_{02}$ ) are 0.889, 0.931 and 0.891, respectively, while in Algorithm 2 (to generate one sample of $\Theta_{1}$ ), acceptance rates are, respectively, 0.990, 0.993 and 0.997. In Algorithm 3 (to generate one sample of $Y_{01}$ ), we have chosen the truncation point $c=0.3,0.6$ and 1.4, respectively, for $\alpha=1.2,1.5$ and 1.8, where we have used the criterion $D_{\mathrm{KS}}(\Delta, c) /\left(\Delta \cdot p_{3}(\Delta, c)\right)$, as in Section 4.3. With the choice of truncation point $c$, acceptance rates in Algorithm 3 are 0.831, 0.588 and 0.276. Even in the case $\alpha=1.8$ with the lowest acceptance rate 0.276 in Algorithm 3, each sample path can be generated within 0.1 second by Scilab software on a computer with recent regular spec. (The computing time can easily be reduced by employing a low-level language, such as C.)

\section{Concluding Remarks}

We have derived exact transition law between consecutive observations of TS-OU processes of infinite variation as a convolution of three random components; a compound Poisson distribution and two tempered stable distributions, one with stability index in $(0,1)$ and the other with index in $(1,2)$. We have adopted acceptance-rejection sampling techniques to simulate exactly the compound Poisson component and the tempered stable distribution with index in $(0,1)$. For simulation of the tempered stable distribution with index in $(1,2)$, we have presented an approximative acceptance-rejection sampling method of [1] with discussion on the issue of trade-off between distribution error and computing time. Sample paths simulation is significantly more efficient with our explicit transition law and simulation techniques, relative to the known approximative method based on infinite shot noise series representation of tempered stable Lévy processes.

As mentioned in Section 4.3, we could think of several approximative simulation techniques for the tempered stable distribution with stability index in $(1,2)$. It would be interesting to investigate those techniques with a view towards simulation. Also, with the explicit transition density functions of TS-OU processes, it is certainly worthwhile to investigate related statistical issues, such as the local asymptotic behavior of the likelihood ratio statistics, efficient parameter estimation, and 
so on. These topics will be investigated in subsequent papers.

\section{References}

[1] Baeumer, B., Meerschaert, M.M. (2009) Tempered stable Lévy motion and transit superdiffusion, Journal of Computational and Applied Mathematics, to appear.

[2] Barndorff-Nielsen, O.E., Shephard, N. (2001) Non-Gaussian Ornstein-Uhlenbeck-based models and some of their uses in financial economics (with discussion), J. R. Statist. Soc. B, 63(2) $167-241$.

[3] Benth, F.E., Gorth, M., Kufakunesu, R. (2007) Valuing volatility and variance swaps for a non-Gaussian Ornstein-Uhlenbeck stochastic volatility model, Applied Mathematical Finance, 14(4) 347-363.

[4] Brockwell, P.J., Davis, R.A., Yang, Y. (2007) Estimation for nonnegative Lévy-driven Ornstein-Uhlenbeck processes, Journal of Applied Probability, 44(4) 977-989.

[5] Chambers, J.M., Mallows, C.L., Stuck, B.W. (1976) A method for simulating stable random variables, Journal of the American Statistical Association, 71(354) 340-344.

[6] Devroye, L. (2009) Random variate generation for exponential and polynomially tilted stable distributions, ACM Transactions on Modeling and Computer Simulation, 19(4) Article No. 18.

[7] Imai, J., Kawai, R. (2009) On finite truncation of infinite shot noise series representation of tempered stable law, in preparation.

[8] Jongbloed, G., van der Meulen, F.H., van der Vaart, A.W. (2005) Nonparametric inference for Lévy-driven Ornstein-Uhlenbeck processes, Bernoulli, 11(5) 759-791.

[9] Kawai, R., Masuda, H. (2009) Exact simulation of finite variation tempered stable OrnsteinUhlenbeck processes, under review.

[10] Masuda, H. (2004) On multidimensional Ornstein-Uhlenbeck processes driven by a general Lévy process, Bernoulli, 10(1) 1-24.

[11] Michael, J.R., Schucany, W.R., Haas, R.W. (1976) Generating random variates using transformations with multiple roots, The American Statistician, 30, 88-90. 
[12] Rosiński, J. (2001) Series representations of Lévy processes from the perspective of point processes, In: Lévy Processes - Theory and Applications, Eds. Barndorff-Nielsen, O.-E., Mikosch, T., Resnick, S.I., Birkhäuser, 401-415.

[13] Rosiński, J. (2007) Tempering stable processes, Stochastic Processes and their Applications, 117(6) 677-707.

[14] Sato, K. (1999) Lévy processes and infinitely divisible distributions, Cambridge University Press.

[15] Sun, S., Zhang, X. (2009) Empirical likelihood estimation of discretely sampled processes of OU type, Science in China Series A: Mathematics, 52(5) 908-931.

[16] Tweedie, M.C.K. (1984) An index which distinguishes between some important exponential families, In: Statistics: Applications and New Directions: Proc. Indian Statistical Institute Golden Jubilee International Conference (eds. J. Ghosh and J. Roy) 579-604.

[17] Zhang, S., Zhang, X. (2008) Exact simulation of IG-OU processes, Methodology and Computing in Applied Probability, 10(3) 337-355.

[18] Zhang, S., Zhang, X. (2009) On the transition law of tempered stable Ornstein-Uhlenbeck processes, Journal of Applied Probability, 46(3) 721-731.

[19] Zolotarev, V.M. (1986) One-Dimensional Stable Distributions, American Mathematical Society, Providence, RI. 

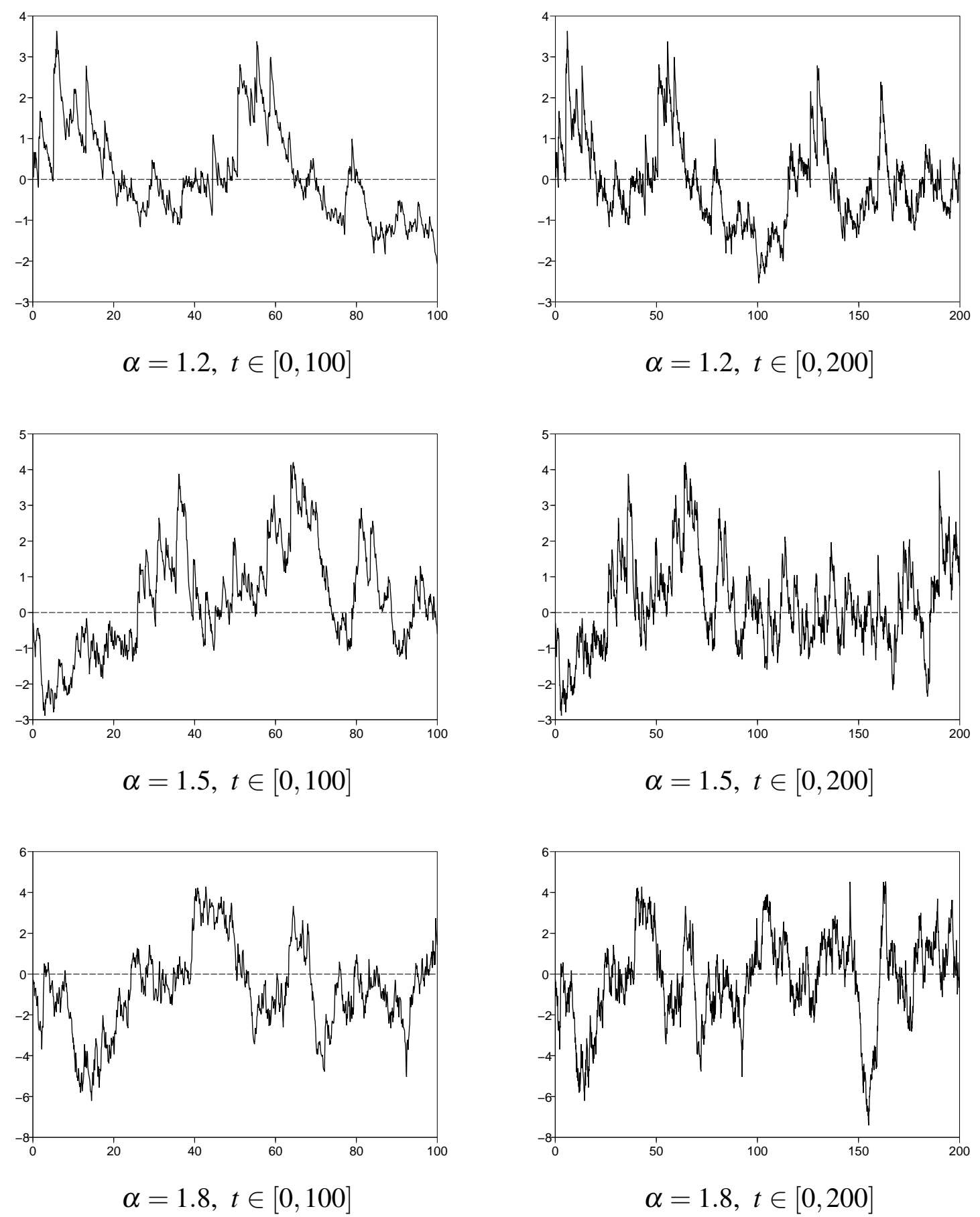

Figure 2: Typical sample paths of tempered stable Ornstein-Uhlenbeck processes through exact simulation algorithm. The horizontal dotted lines indicate $X_{0}=0\left(=\lim _{t \uparrow+\infty} \mathbb{E}\left[X_{t}\right]\right)$. 


\section{List of MI Preprint Series, Kyushu University}

The Global COE Program

Math-for-Industry Education \& Research Hub

MI

MI2008-1 Takahiro ITO, Shuichi INOKUCHI \& Yoshihiro MIZOGUCHI

Abstract collision systems simulated by cellular automata

MI2008-2 Eiji ONODERA

The intial value problem for a third-order dispersive flow into compact almost

Hermitian manifolds

MI2008-3 Hiroaki KIDO

On isosceles sets in the 4-dimensional Euclidean space

MI2008-4 Hirofumi NOTSU

Numerical computations of cavity flow problems by a pressure stabilized characteristiccurve finite element scheme

MI2008-5 Yoshiyasu OZEKI

Torsion points of abelian varieties with values in nfinite extensions over a padic field

MI2008-6 Yoshiyuki TOMIYAMA

Lifting Galois representations over arbitrary number fields

MI2008-7 Takehiro HIROTSU \& Setsuo TANIGUCHI

The random walk model revisited

MI2008-8 Silvia GANDY, Masaaki KANNO, Hirokazu ANAI \& Kazuhiro YOKOYAMA Optimizing a particular real root of a polynomial by a special cylindrical algebraic decomposition

MI2008-9 Kazufumi KIMOTO, Sho MATSUMOTO \& Masato WAKAYAMA

Alpha-determinant cyclic modules and Jacobi polynomials 
MI2008-10 Sangyeol LEE \& Hiroki MASUDA

Jarque-Bera Normality Test for the Driving Lévy Process of a Discretely Observed Univariate SDE

MI2008-11 Hiroyuki CHIHARA \& Eiji ONODERA

A third order dispersive flow for closed curves into almost Hermitian manifolds

MI2008-12 Takehiko KINOSHITA, Kouji HASHIMOTO and Mitsuhiro T. NAKAO

On the $L^{2}$ a priori error estimates to the finite element solution of elliptic problems with singular adjoint operator

MI2008-13 Jacques FARAUT and Masato WAKAYAMA

Hermitian symmetric spaces of tube type and multivariate Meixner-Pollaczek polynomials

MI2008-14 Takashi NAKAMURA

Riemann zeta-values, Euler polynomials and the best constant of Sobolev inequality

MI2008-15 Takashi NAKAMURA

Some topics related to Hurwitz-Lerch zeta functions

MI2009-1 Yasuhide FUKUMOTO

Global time evolution of viscous vortex rings

MI2009-2 Hidetoshi MATSUI \& Sadanori KONISHI

Regularized functional regression modeling for functional response and predictors

MI2009-3 Hidetoshi MATSUI \& Sadanori KONISHI

Variable selection for functional regression model via the $L_{1}$ regularization

MI2009-4 Shuichi KAWANO \& Sadanori KONISHI

Nonlinear logistic discrimination via regularized Gaussian basis expansions

MI2009-5 Toshiro HIRANOUCHI \& Yuichiro TAGUCHII

Flat modules and Groebner bases over truncated discrete valuation rings 
MI2009-6 Kenji KAJIWARA \& Yasuhiro OHTA

Bilinearization and Casorati determinant solutions to non-autonomous $1+1$ dimensional discrete soliton equations

\section{MI2009-7 Yoshiyuki KAGEI}

Asymptotic behavior of solutions of the compressible Navier-Stokes equation around the plane Couette flow

MI2009-8 Shohei TATEISHI, Hidetoshi MATSUI \& Sadanori KONISHI

Nonlinear regression modeling via the lasso-type regularization

MI2009-9 Takeshi TAKAISHI \& Masato KIMURA

Phase field model for mode III crack growth in two dimensional elasticity

MI2009-10 Shingo SAITO

Generalisation of Mack's formula for claims reserving with arbitrary exponents for the variance assumption

MI2009-11 Kenji KAJIWARA, Masanobu KANEKO, Atsushi NOBE \& Teruhisa TSUDA Ultradiscretization of a solvable two-dimensional chaotic map associated with the Hesse cubic curve

\section{MI2009-12 Tetsu MASUDA}

Hypergeometric T -functions of the q-Painlevé system of type $E_{8}^{(1)}$

MI2009-13 Hidenao IWANE, Hitoshi YANAMI, Hirokazu ANAI \& Kazuhiro YOKOYAMA A Practical Implementation of a Symbolic-Numeric Cylindrical Algebraic Decomposition for Quantifier Elimination

MI2009-14 Yasunori MAEKAWA

On Gaussian decay estimates of solutions to some linear elliptic equations and its applications

MI2009-15 Yuya ISHIHARA \& Yoshiyuki KAGEI

Large time behavior of the semigroup on $L^{p}$ spaces associated with the linearized compressible Navier-Stokes equation in a cylindrical domain 
MI2009-16 Chikashi ARITA, Atsuo KUNIBA, Kazumitsu SAKAI \& Tsuyoshi SAWABE Spectrum in multi-species asymmetric simple exclusion process on a ring

MI2009-17 Masato WAKAYAMA \& Keitaro YAMAMOTO

Non-linear algebraic differential equations satisfied by certain family of elliptic functions

MI2009-18 Me Me NAING \& Yasuhide FUKUMOTO

Local Instability of an Elliptical Flow Subjected to a Coriolis Force

MI2009-19 Mitsunori KAYANO \& Sadanori KONISHI

Sparse functional principal component analysis via regularized basis expansions and its application

MI2009-20 Shuichi KAWANO \& Sadanori KONISHI

Semi-supervised logistic discrimination via regularized Gaussian basis expansions

MI2009-21 Hiroshi YOSHIDA, Yoshihiro MIWA \& Masanobu KANEKO

Elliptic curves and Fibonacci numbers arising from Lindenmayer system with symbolic computations

MI2009-22 Eiji ONODERA

A remark on the global existence of a third order dispersive flow into locally Hermitian symmetric spaces

MI2009-23 Stjepan LUGOMER \& Yasuhide FUKUMOTO

Generation of ribbons, helicoids and complex scherk surface in laser-matter Interactions

MI2009-24 Yu KAWAKAMI

Recent progress in value distribution of the hyperbolic Gauss map

MI2009-25 Takehiko KINOSHITA \& Mitsuhiro T. NAKAO

On very accurate enclosure of the optimal constant in the a priori error estimates for $H_{0}^{2}$-projection 
MI2009-26 Manabu YOSHIDA

Ramification of local fields and Fontaine's property (Pm)

MI2009-27 Yu KAWAKAMI

Value distribution of the hyperbolic Gauss maps for flat fronts in hyperbolic three-space

MI2009-28 Masahisa TABATA

Numerical simulation of fluid movement in an hourglass by an energy-stable finite element scheme

MI2009-29 Yoshiyuki KAGEI \& Yasunori MAEKAWA Asymptotic behaviors of solutions to evolution equations in the presence of translation and scaling invariance

MI2009-30 Yoshiyuki KAGEI \& Yasunori MAEKAWA

On asymptotic behaviors of solutions to parabolic systems modelling chemotaxis

MI2009-31 Masato WAKAYAMA \& Yoshinori YAMASAKI

Hecke's zeros and higher depth determinants

MI2009-32 Olivier PIRONNEAU \& Masahisa TABATA

Stability and convergence of a Galerkin-characteristics finite element scheme of lumped mass type

MI2009-33 Chikashi ARITA

Queueing process with excluded-volume effect

MI2009-34 Kenji KAJIWARA, Nobutaka NAKAZONO \& Teruhisa TSUDA

Projective reduction of the discrete Painlevé system of type $\left(A_{2}+A_{1}\right)^{(1)}$

MI2009-35 Yosuke MIZUYAMA, Takamasa SHINDE, Masahisa TABATA \& Daisuke TAGAMI Finite element computation for scattering problems of micro-hologram using DtN map 
MI2009-36 Reiichiro KAWAI \& Hiroki MASUDA

Exact simulation of finite variation tempered stable Ornstein-Uhlenbeck processes

MI2009-37 Hiroki MASUDA

On statistical aspects in calibrating a geometric skewed stable asset price model

MI2010-1 Hiroki MASUDA

Approximate self-weighted LAD estimation of discretely observed ergodic OrnsteinUhlenbeck processes

MI2010-2 Reiichiro KAWAI \& Hiroki MASUDA

Infinite variation tempered stable Ornstein-Uhlenbeck processes with discrete observations 